\title{
The holobiont self: understanding immunity in context
}

\author{
Tamar Schneider $^{1}$ (D)
}

Received: 16 December 2020 / Accepted: 26 July 2021 / Published online: 9 August 2021 (C) Springer Nature Switzerland AG 2021

\begin{abstract}
Both concepts of the holobiont and the immune system are at the heart of an ongoing scientific and philosophical examination concerning questions of the organism's individuality and identity as well as the relations between organisms and their environment. Examining the holobiont, the question of boundaries and individuality is challenging because it is both an assemblage of organisms with physiological cohesive aspects. I discuss the concept of immunity and the immune system function from the holobiont perspective. Because of the host-microbial close relations of codependence and interdependence, the holobiont is more often than not confused with the host, as the host is the domain in which this entity exists. I discuss the holobiont unique ecological characteristics of microbial assemblages connected to a host in a network of interactions in which the host is one of the organisms in the community but also its landscape. Therefore, I suggest viewing the holobiont as a host-ecosystem and discuss the implication of such a view on the concept of immunity and the meaning of protection. Furthermore, I show that viewing the holobiont as a host ecosystem opens the possibility of using the same ecological definition of boundaries and immunity dealing with an ecological system. Thus, the holobiont's boundaries and immunity are defined by the persistence of its complex system of interactions integrating existing and new interactions. This way of thinking presents a notion of immunity that materializes as the result of the complex interdependence relations between the different organisms composing the holobiont similar to that of an ecosystem. Taking this view further, I discuss the notion of immunogenicity that is ontologically heterogeneous with various causal explanations of the processes of tolerance and targeted immune response. Finally, I discuss the possible conceptualization of already existing and new biomedical practices.
\end{abstract}

Tamar Schneider

Tamisch0106@gmail.com

1 Cohn Institute for History and Philosophy of Science and Ideas, Humanities Faculty, Tel Aviv

University, Ramat Aviv, 6997801 Tel Aviv, Israel 
Keywords Holobiont $\cdot$ Host ecosystem $\cdot$ Ecological community $\cdot$ Immunity · Persistence and stability of interactions

\section{Introduction}

The holobiont is an entity of a host organism living with close and intimate microbial communities (i.e., microbiomes) and the immune self refers to the host's immunological identity. Furthermore, the holobiont and the immune self are both at the heart of an ongoing scientific and philosophical examination concerning questions on the relations between organisms and their environment. Immunity and the immune self are concepts used in the demarcation of a physiological individuality with distinct boundaries and physiological identity (Pradeu, 2016, 2019; Tauber, 2016, 2017). Examining the holobiont, the question of physiological boundaries and individuality is challenging because it is both an individual and an assemblage of organisms with physiological cohesive aspects. Some forms of holobionts are considered individuals depending on the levels of cohesion with their microbes, while others are viewed as an ecological community (Douglas \& Werren, 2016; Lloyd \& Wade, 2019; Queller \& Strassmann, 2016; Skillings, 2016; Suárez \& Stencel, 2020). I discuss the concept of immunity and the immune system function from the holobiont perspective. Because of the host-microbial close relations of interdependence, the holobiont is more often than not confused with the host, as the host is the domain in which this entity exists. For example, the host's immune system depends on the interactions with the microbial communities for its development and function. Also, the death of the host affects the holobiont's persistence. Therefore, further analysis and perhaps an alternative view on immunity can help clarify the confusion between the host and the holobiont, which are not the same but very much entangled.

The discussion on the holobiont as an ecological community addresses two aspects of the holobiont: one is the assemblage of host and microbes that allows fuzzy boundaries and interchangeable composition and interactions between organisms. Here, there should be a distinction between the host and the microbes as separate individuals with their internal organization and immunity. The second aspect is of a host that in some respect is a heterogeneous entity individualized by its immune system that protects or maintains its internal order, and distinctiveness of its symbionts from the rest of its microbial environment (Pradeu, 2016, 2019). The latter aspect considers only the symbionts with high fidelity and cohesion as part of the physiological host. However, because of the dynamic nature of such interactions in some cases the distinction between the host and what should be considered the holobiont is less clear. ${ }^{1}$

\footnotetext{
1 In cases of endosymbionts such as the mitochondria, the inclusion as part of the host is clearer. But in other cases, such as the obligatory symbionts $V$. fischeri and the squid is less clear because in nature the symbiont is considered obligatory, but in the lab, the squid can be cultivated without the symbiont (Koch et al., 2020). Also, the bacteria apparently can exist outside the squid and do not need the light organ for their survival (Skilling 2016).
} 
This discourse confuses the holobiont with the host in one aspect (the physiological individual) and forcing a distinction that is not the case on the other (i.e., an ecological community). One suggestion for dealing with this confusion is suggested by Suárez and Stencel's (2020) ontological analysis of a part-dependent biological individual. They offer to view the holobiont as an individual from the host perspective and as an ecological community from the microbial perspective. While I agree with their analysis showing the asymmetry between host and microbes, I still argue that the physiological unit of host individual as Suárez and Stencel (2020) put it needs further discussion into its heterogeneity and lack of symmetry when discussing immunity. Thus, the ontological part-dependent analysis maintains the notion of a physiological unit that holds heterogeneous interactions and asymmetry. Therefore, I suggest having an alternative view of what is a physiological unit through an ecological perspective on individuality.

I suggest a solution for this host/holobiont entanglement by discussing an alternative perspective on immunity and physiological boundaries. The holobiont is a unique ecological entity because of its structure of a host and microbial relations in which the host is one of the organisms in the community but also its landscape. Furthermore, this unique ecological community has different size and temporal scales mutually interacting in heterogeneous and interdependence relationships. Therefore, I suggest viewing the holobiont as a host ecosystem using ecological concepts in the examination of boundaries and immunity. ${ }^{2}$ Thus, viewing the holobiont and its immunity from an ecological perspective as a host-ecosystem. This way of thinking presents a notion of immunity from an ontological heterogeneity perspective as the complex system of many interactions between entities that contributes to the overall well-being and capacity of the ecological system (i.e., the holobiont). In this view, immunity is the result of the interactions between the organisms in an ecological entity that is the holobiont. ${ }^{3}$

First, I look at the conceptualization challenges of this unique entanglement of the host and the holobiont suggesting the view of the holobiont as the host ecosystem. Then, I argue that the holobiont's immunity, viewed from an ecological perspective, consists in the persistence and stability of the complex system of interactions between the organisms composing the holobiont in their environment. Thus, the immunological concept of the immune self should represent this notion. Immunity in this regard does not belong to the single organism rather the immunity materializes through assemblages of organisms and their interactions. Therefore, the immune self should refer to the holobiont i.e., the Holobiont Self. Next, I discuss

\footnotetext{
${ }^{2}$ Here, I suggest viewing boundaries through the scope of interactions and immunity in terms of stability and persistence. I acknowledge that these concepts are contested in ecology as the former can have two meanings, one is a constant state and the other as stability in between non-stable states (Millstein $2020 \mathrm{a}, \mathrm{b})$. Also, persistence can mean returning to the same state as before perturbation or returning to a trajectory of functions with changes. I believe that using these concepts in a physiological context can benefit both fields if being scrutinized by their differences and similarities in frameworks, assumptions, and background beliefs.

3 Respectfully, this means that immunity also excludes the entities that do not promote stability. This exclusion is done by the immune cells' response and the bacterial interactions. This role might fit with the traditional way of thinking about immunity as a system of protection.
} 
the implication of the holobiont self and immunity as a process on the meaning of boundaries. I argue that the immunological boundaries of the holobiont need to be examined by the processes of interactions and integration (not by discrimination and separation). Thus, the immunological boundaries are the holobiont's outlines (not its border or de-fence), which develop and change throughout the host's life. Finally, I discuss some possible objections to my view which look at the biomedical practices and conclude with future implications to medical and immunological studies.

\section{The unique features of the ecological community that is the holobiont}

The notion of the holobiont as a heterogeneous biological entity raises the questions of what it includes in terms of part-whole relations (e.g., groups of organisms, host and symbionts, host and microbiomes, etc.); what are the factors that determine its physiological boundaries and its organismal identity in terms of inside and outside (e.g. genes, molecular bindings, territory, ecological niche, etc.); and how to reconcile the fact that one biological entity (the holobiont) can be composed of other biological entities (i.e., symbionts, and endosymbionts) but does not, in most cases, depend on their specific existence for survival (Douglas \& Werren, 2016; Moran \& Sloan, 2015; Skillings, 2016; Triviño \& Suarez, 2020). For example, the composition of gut bacteria can change, yet, the host will not perish. However, if the host loses all its gut bacteria and cannot regain new ones it may not survive (McFallNgai et al., 2013).

The holobiont is a community composed of interactions between organisms of very different sizes and life cycles, of small tiny microorganisms constructed around a host organism. That alone presents a dilemma: on the one hand, we have a particular host organism we can distinguish from other hosts organisms, and address the host as a physiological individual (Pradeu, 2016). On the other hand, this host is connected in symbiotic crucial interactions with interchangeable microbial communities of which it cannot live without. The host's entanglement with the microbial communities is with no clear distinction between symbiotic microbes and environmental (i.e., nonresident microbes), makes it harder to delineate the immunological boundaries. This dilemma is at the heart of the debates around the concept of the holobiont, which entails individuality from the host perspective and ecological community from the microbial perspective (Lloyd \& Wade, 2019; Suárez \& Stencel, 2020; Triviño \& Suarez, 2020).

To make the symbiotic/environmental microbial distinction, different claims are made regarding the nature of the interactions and relations between the organisms composing the holobiont. Different claims regarding the individuality of the holobiont center on the evolutionary perspective examining issues such as co-evolution, hereditary, reproduction, and natural selection (Douglas \& Werren, 2016; Lloyd, 2017; Lloyd \& Wade, 2019; Roughgarden et al., 2018; Skillings, 2016; Theis et al., 2016). More recent analyses by Lloyd and Wade (2019) suggested a more pluralistic view acknowledging the multiple shapes and variation of host-microbial symbiosis, pointing to a spectrum of interactions included in the holobiont concept stretched from Demibiont to Euholobiont. Another pluralist view by Suárez and Stencel 
(2020) ontological analysis of the holobiont of part-dependent definition argues that the holobiont is a biological individual from the host standpoint and an ecological community from that of the microbes.

Most of these claims, however, do not address the question of boundaries from an immunological perspective despite the latter's significant conceptual role in physiological boundaries delineation and individualization (Pradeu, 2016, 2018, 2019). To address the immunological issue, Thomas Pradeu (2016) makes the distinction between the physiological individual and the evolutionary individual. He argues that when thinking about the evolutionary individual, the evolutionary unit (whether interactor, reproducer, or Darwinian individuals) can also be the unit of living but not necessarily (Pradeu, 2016). Thus, the physiological individual relates to the boundaries and degrees of cohesion while the evolutionary individual relates to variation, heritability, reproduction processes, and differential fitness (Pradeu, 2010, 2011b, 2016). This distinction, according to Pradeu, clarifies the understanding that when thinking about definitions of the organism or unit of living, the discussion is of the physiological organization that can include the collaboration between different evolutionary units (Pradeu, 2016). This distinction, however, is still confusing because of the ongoing debate on the definition of the unit of selection, and the question of what should be part of the unit versus its environmental context (Lloyd, 2001, 2017). In the next section, I show that there is also confusion regarding the physiological and immunological definitions of boundary delineation and the pathogenic/non-pathogenic distinction (also thought of as self/non-self discrimination or in its newer version as the persistence of the immunological identity).

Thinking about the holobiont in its most descriptive form as an ecological entity of a host-organism and microorganisms, is on the one hand, helpful because it potentially allows capturing characteristics such as the heterogeneity of interactions, fuzzy boundaries, and the holobiont being ephemeral. But, on the other hand, the holobiont is closely constructed around, outside, and inside a host-organism, with the microbes being part of the host's developmental processes, the immune system, digestive system, and possibly the endocrine and nervous system (Gilbert et al., 2012). Considering this structure, the questions of inside/outside of the holobiont are entangled with questions of inside/outside of the host-organism. Particularly, when considering the holobiont's features such as (1) the holobiont is closely structured around the host-organism; (2) it is composed of various microbial communities that are also facilitating the host's developmental processes as scaffolds (Chiu \& Eberl, 2016; Griesemer, 2016); and (3) it dies approximately to when the host dies (or changes into something else, i.e., a different type of ecological community that is no longer the holobiont). These unique features can also portray the ecological nature of the holobiont from the host perspective as a host ecosystem, i.e., the host and microbial biotic interactions and abiotic interactions of nutrients, metabolites, which are also the host physiological systems.

For example, the holobiont of the Hawaiian bobtail squid and the bacteria Vibrio fischeri can be described as an ecological community of the squid and bacteria. But when looking at the molecular level of interactions and to the evolutionary history of the relationship, it is also an ecological system in which the $V$. fischeri that lives in the squid's light organ changes the latter's entire biochemistry (Koch et al., 2020; 
Lloyd \& Wade, 2019). ${ }^{4}$ The boundaries in this unique community are fuzzy, but also, follow the host closely. More so, the holobiont as an ecological system transitions into a different system of decomposers after the host dies, and the host's wellbeing and survival depended on its interactions with its microbial communities. Furthermore, just as the squid is the vibrio's ecological niche so does the microbiome in the sand are the squid's microbial niche, and the ecological persistence of the SquidVibrio holobiont is entangled with that of the sand microbiomes. Thus, the Squid population is also a part of the microbial ecological system or ecosystem. The existence, persistence, and life cycle of the single holobiont is proximate and delimited by the host life cycle and persistence, but also depend on the microbial communities in the larger ecosystem where the holobiont species population lives.

Suárez and Stencel (2020), ontological analysis of the part-dependent definition of the biological individual is based on the presupposition that for the definition of individuality one needs to have relations of dependence between its different elements. They argue that in the part dependent definition such dependency need not be symmetrical. However, their discourse on the host-dependent view of the holobiont as an individual is mainly organismal (physiological) and evolutionary centered. Thus, individuality is characterized by relations of dependence, a "cohesive, welldelineated and countable unit from the perspective of some elements but not others" (Suárez \& Stencel, 2020, p. 1318 emphasis added). In contrast to this view, I wish to look at the ecological perspective of individuality emphasizing processes of various interactions and their interdependence (Millstein, 2018, 2020a). In this ecological view, ecological systems are considered individuals because of their complex system of interactions and interdependence between organisms. Also, such an ecological individual does not preclude the understanding of different levels of interdependence and dependence between different entities.

In ecology, there are two distinguished but connected approaches of ecosystem ecology and ecological community. The community ecology approach emphasizes the interactions between organisms looking at species populations, their interactions (role function), and distribution (biotic community). The ecosystem ecology approach follows the abiotic components examining matter and energy flow and flux circulating in the system including dead organisms and nutritive. In her paper "Lobopodian concept of Land Community," Roberta Millstein (2018) discusses past and present ecologists who combine aspects of the concept of ecological community with aspects of the concept of ecosystem. The main presuppositions of this combining approach are (1) there is a system of interdependence between species populations in an ecological community through trophic relations (i.e., predator-prey, decomposers, parasites, cross-feeding, etc.,); (2) There are complex interrelations between biotic systems such as interactions between species-populations, and physical systems such as light, acidity, levels of oxygen, spatial conditions, which shapes

\footnotetext{
${ }^{4}$ Koch et al. show that the absence of $\mathrm{V}$. fischeri in the light organ significantly affects $\sim 25 \%$ of the hemolymph (the fluid analogous to the blood in vertebrates) metabolites of an adult male E. scolopes. They sampled the metabolites in the squid's hemolymph three times over a $24 \mathrm{~h}$ diel cycle to show the correlations between levels of these biomolecules and the rhythms of symbiont presence. The correlations suggest that the presence of a single species of symbiont can modulate the daily rhythms of small molecules circulating throughout its host. (Koch et al., 2020).
} 
the systems of matter and energy flow as well as the physical niche; and (3) There is a clear distinction in ecology between the Ecosystem, which is the ecological entity, and models of ecosystem processes (Millstein, 2018).

Furthermore, the ecosystem entity is conceived as a system of interrelations of interdependence between the biotic and abiotic components through their various processes of interactions. Interactions in this case refer to both between organisms and between organisms and physical components of the environment. The meaning of function in ecology is the description of these interactions as processes of state changes in the system (Jax, 2005). Thus, the interactions as functions can be categorized in terms of role functions such as predators, decomposers, and also some can be considered as ecosystem's services such as the decomposers (Jax, 2005). Ecosystems are in a constant state of fluctuation with the stability understood as the ability to return to the state of equilibrium and maintain the trajectories of the complex whole of the system's functions, i.e., the functions or network of interactions are in homeorhetic stability (Jax, 2005; Millstein, 2018, 2020b). Considering this combined community-ecosystem concept, I suggest comprising all the ecological communities (biotic and abiotic interactions between microbes in the microbiome, and interactions between the host and the microbes) within an ecosystem entity, which is the host. Thus, the host-ecosystem is the complexity of a host and microbial interactions, which also involve abiotic interactions (such as metabolism and vitamin synthesis) to maintain and facilitate the host's physiological system. Thus, looking at the individuality aspect from an ecological perspective as an ecological individual, i.e., the host-ecosystem.

Like Suárez and Stencel (2020) or Lloyd and Wade (2019), I argue that because of this unique structure, the definition of the holobiont as an ecological community does not solve the dilemma of how to understand the host perspective of the holobiont. However, I further claim that when trying to distinguish the question of the holobiont physiological boundaries from the question of the host immunological boundaries it becomes clear that both perspectives of the host-individuality and the microbiome-ecology are very much entangled, thus, either is insufficient in providing an immunological or physiological account for the holobiont. For example, thinking about the holobiont of human and gut microbiome: on the one hand, we have a biological individual that includes the host interacting with microbes first introduced at birth. But on the other hand, we have ecological communities developing in the host as the host becomes the microbiomes environmental niche. Furthermore, the microbial environmental niches are active, also involving interactions with the host's immune cells (Baquero et al., 2021). In other words, I ask about the host role within the holobiont ecological community (i.e., understanding the host and its immunity from an ecological perspective). Is the host considered another organism in such a community? and if this is the case, then the host is clearly in a different position from the other interacting bacteria as Suárez and Stencel (2020) show, and as I have discussed above. Here, I suggest looking at the immunology of the host by considering also how the set of microbes of its microbiome contribute to it, and how they may change the host's biological nature (biochemistry, history, etc.). In doing so, I would be combining the best of the ecological interpretation that Suárez and 
Stencel preclude exclusively to the microbes, with the best of the individuality view that they attribute to the host.

This ecological view of the holobiont as a host-ecosystem encompasses both the host and the microbes as opposed to the view that distinguishes the individualorganismal nature of the holobiont from the communal ecological aspect. Thus, in some respect, the host is the microbial niche but in some respects, the microbial communities are the host's niche. ${ }^{5}$ Including the host perspective in the ecological part-dependent view means that the host-individuality aspect of the holobiont is more likely to be of an ecological nature. In other words, the notion of an organism is also of an ecological nature of a complex interactions system that is open non-stable and fluctuating. Furthermore, the notion of individuality in this regard is closer to the notion of an ecosystem as an individual (Huneman, 2014; Millstein, 2018, 2020a; Odenbaugh, 2007, 2010). Thus, changing the framework of the host perspective of the holobiont from that of physiological to that of ecological and immunological, the conceptualization of immunity changes as well to be within ecological perspective, i.e., eco-immunity (Tauber, 2016). This move also changes the perspective on the immune identity from a property of the host to that of a system of interactions that materializes as the host-ecosystem, i.e., the holobiont. In this view, immunity is a process of interactions and intra-actions ${ }^{6}$ between entities, and therefore, cannot be attributed exclusively to the host irrespectively of its interactions with the microbes (Nicholson \& Dupré, 2018).

I introduce the term "intra-action" in recognition of the organisms' ontological inseparability from their close and intimate environment. Interaction relies on the metaphysics of individualism that presupposes the independent existence of autonomous entities prior to their interactions with other entities and their environment. The notion of intra-actions presupposes the entities' materialization is through the iteration of interactions with other entities. Taking this concept to the holobiont and immunity, the host and microbial intra-actions are the iterations of biotic and abiotic interactions materializing into a host-ecosystem. Some of these interactions are between the host's cells (in particular the immune cells) with the microbes that build the host's immunity and help in the development and function of the host's physiological systems. The intra-actions are the interactions between entities (i.e., host and microbes, and between hosts and microbes) and between entities and the environment that are interdependent and constituting each other, but also at the same time differentiated entities. In other words, the microbial cells and body cells interact involving their background conditions creating the holobiont such that their actions and characteristics manifest by these interactions (Schneider, 2020a, b). Intra-action means that the interacting cells become the holobiont (i.e., the phenomenon) and as they are taken out of the holobiont their actions and interactions change as well as their characteristics (Barad, 2007).

\footnotetext{
${ }^{5}$ I thank the anonymous reviewer for this clarification of the importance of the host's microbial niche that is shaped and established by the host's behavior, waist, nutritional, and hygienic habits.

${ }^{6}$ Intra-actions are such interactions that materialize to a phenomeon of the interacting entities through iterations of interactions such as a dyad, community, or social construct (Barad 2007; Griesemer 2014).
} 
Furthermore, I argue, the immunity that is the result of interactions and intraaction changes the way we should think about the physiological and immunological boundaries, not in terms of separating borders, but as the outlines that develop and change through integration of interactions. In the following Sects. 3 and 4, I discuss, first the notion of immunity as a process of integration and persistence of interactions, and then the understanding of boundaries in terms of interactions.

\section{The holobiont self: a view of immunity as the system of integration and persistence of interactions}

The immune self metaphor in immunology, still dominant despite conceptual and empirical attempts of replacing it, frames the immune system's role as a discriminatory mechanism of harmful (i.e., pathogenic) from non-harmful (i.e., non-pathogenic) elements in the body. This view in immunology first introduced by the Australian immunologist Macfarlane Burnet in 1949 looks at immunity as 'belonging' to the organism, 'protecting' it from non-self elements. The main critique of the discriminatory self elaborated by Alfred Tauber's pervasive writing is that of the philosophical, or more specific, epistemic unstable ground of the concept of the self together with new evidence of the symbiotic nature of the host interactions with microbes (Gilbert \& Tauber, 2016; Gilbert et al., 2012; Tauber, 2017). ${ }^{7}$ Tauber's alternative approach considers the immune system and immunity from an ecological perspective, which includes immune tolerance responses, and the maintenance of the organism's internal interactions in a cohesive balance distinguished from other environmental interactions (Tauber, 2016, 2017). In a shared paper with Scott Gilbert they write:

The multiple functions of the immune system, especially its ability to achieve states of tolerance for assimilative processes expand the self-defensive role of immunology into a larger "ecological" context of promoting organismal identity through dialogue with both the internal and external environments. In other words, the original clinical orientation of immunology is being supplemented with an expanded ecological orientation where immunology is joining the environmental sciences (Gilbert \& Tauber, 2016, 844 emphases added)

Here, the move from the self-defensive role by discrimination to the defensive role by promoting identity replaces the immune self with the immunological identity. Though looking at the organismal identity as a configuration of its relations with its microbial environment is less prone to essentialism as suggested by Thomas Pradeu, it remains on the same conceptual ground connecting immunity with the individual and the process of individualization (Pradeu, 2019; Pradeu

\footnotetext{
7 Tauber also follows the theoretic and conceptual work of one of the pioneer researchers in immunology Elie Metchnikoff, who discovered phagocytosis, as an alternative view to Burnet's discriminatory self. Tauber describes Matchnikoff's view of immunological function in a more ecological perspective also placing inflammation as part of the organism's developmental processes (Tauber 1994; Tauber and Chernyak 1991).
} 
\& Carosella, 2006). The conceptual work done by the notion of immunological identity is the ability to establish a coherent individual and find one causal explanation of the mechanisms of an immune response, i.e., immunogenicity (Pradeu \& Carosella, 2006; Pradeu, 2016, 2018, 2019). Thus, the meaning of immunological protection changes from the protection of the self from non-self to the protection of the organism's coherent/stable identity.

By connecting protection with identity, the immune function is no different from the traditional self/non-self discriminatory function because of the emphasis on the immune entity and its identity as a trigger for immune response (i.e., tolerance or targeting). In other words, the immune self in its new meaning as immunological identity, i.e., what the system is, is connected to an explanation of the immune function of what the system does (i.e., immunogenicity). However, this connection is based on the presupposition regarding the meaning and interpretation of the notion of protection.

In the immune identity framework, the thought is that to protect an entity $\mathrm{X}$ from harm/disturbance we need to identify X's identity as an indication of an approximate desired state of stability. This supposition looks at the function of protection by maintaining the stability or cohesion of the identity. For example, in Pradeu's continuity theory, the discontinuity of interactions is considered harmful because it violates the coherent process of immunological identity maintained in homeostasis (Pradeu, 2016, 2018). Both of the notions of self discriminatory and immunological identity are looking for a generalization of the causal explanation of the immune response. Both maintain the immune function as stemming from some sort of a cohesive identity that needs protection by discrimination and distinction (Pradeu, 2016, 2018, 2019; Gilbert \& Tauber, 2016; Tauber, 2017). The main important difference between the immune-self and the immune-identity is that the former considers protection in separating the self from the environment while the latter looks at the self-identity integration with the environment (Gilbert \& Tauber, 2016).

Tauber and Pradeu suggest an alternative understanding of the protective function of immunity that is by integrating rather than separating. Here, the immunological identity is dynamic and changes with the process of integration, and the protective role portrait by the discriminating among interactions and new encounters, which allows the development and change of the self/identity (Gilbert \& Tauber, 2016; Pradeu, 2019; Tauber, 2017). In this framework, the boundaries of the immunological entity that needs protection are outlines that go through changes by introducing new interactions from the environment. The protection is by the ability to integrate and not by the ability to separate. Though agree with the notion of integration, I wish to question the discriminatory role assigned to the immune system. My point here is not to argue that the immunological identity is inconsequential in the process of persistence and stability of the host organism. What I claim is that the immunological identity should not be taken as the focal causal explanation for the various immune responses (i.e., immunogenicity).

The question I wish to raise here is why should identity define protection? In other words, I wish to introduce an alternative way of thinking about the meaning of immunological protection or immunity. My suggestion has two parts which the first 
one is in line with Pradeu's notion of the immunological interactions as the basis for establishing identity and a certain kind of individuality. However, argue that the notion of interactions as the basis for identity or individuality does not entail a discriminatory function. Thus, I suggest a different perspective on immunity that is not protection by discrimination but protection by interactions and intra-action that contribute to the persistence through environmental changes and development. Here I wish to push further the notion of eco-immunity to an ontological heterogeneity way of thinking and suggest various contingent processes of immunogenicity that are not part of one possible explanation. Thus, the view I am offering here is that there is no one generalized scientific explanation of the activation of an effector immune response in the form of discriminatory mechanisms, but rather many various ecological interactions connected in an interdependence ecological system of interactions to maintain a developmental continuum.

Thinking of integration, we do not need to change the immunological identity suggested by Pradeu (2016) as the relational and dynamic continuity identity, only the conceptualization of its function. Here, there is still discrimination of those interactions not integrated, but the focus is different. Immunity is the process of integration, involving more than one organism. For example, the immunity of the squid-vibrio holobiont involves both organisms (probably more when looking at the female's microbiome) and the latter is also connected to the immunity of the holobiont Squid-Vibrio species-population in their environmental niche. The same goes for the gut microbiome and the host as an ecological system with their different levels of interactions and interdependence and their larger environmental species-population context. This view is close to Tauber and Gilbert's view of eco-immunity but with the perspective in which the immune system is less a negotiator with a "reactive spectrum of tolerance and rejection" (846), rather integrated as part of the inner ecological interactions.

Protection of identity views immune function in maintaining the internal organization in a stable state by the process of homeostasis (Pradeu, 2018, 2019). My suggestion of protection of the host-ecosystem in ecological terms with the understanding that it is a system of constant trajectories of homeostasis due to developmental and also environmental fluctuation. Thus, the meaning of stability is the returning to balance that is on a continuum of a trajectory of integration of same and new interactions creating a dynamical functioning system (Jax, 2005; Millstein, 2018, 2020a). Viewing immunity of an integrated fluctuating system of interactions is better framed in terms of homeorhesis. Homeorhesis is the property of a system that can return to a trajectory of patterns of interactions after perturbations or changes, which is different from that of homeostasis that describes the system's stability of returning to balance (Waddington, 1957). When looking at immunity, environmental and developmental perturbations introduce changes to the host, the immune cells, and to the microbial composition and function introducing a new state of stability. Thus, immunity is the homeorhetic ability of this ensemble to integrate entities to persist in a way that maintains the system's stability of the complex of the new 
and old interactions integrated into a functioning system. ${ }^{8}$ The continuity here is between the historical dynamics of interactions and possible future trajectories of interactions in such a way that enables the persistence of the host and microbial ecological complex system (its existence, development, and potential of reproduction).

Furthermore, theorizing immunity as a property of the organism with the main objective of determining its immunological identity considers the immune system as the causal effective entity. However, by viewing immunity as a process of interactions' integration and persistence the mechanism of discrimination of tolerance and targeted response is only one pattern of interactions among others that also included in the holobiont's immunity, such as the microbial web of interactions such as crossfeeding, molecular signaling, lateral gene transfer, quorum sensing, and other types of interactions (Konopka, 2009; O'Malley, 2014). This perspective presupposes the ontological heterogeneity of the causality and involvement of more than one entity in the process of immunity (Schneider, 2020a). For example, the microbial metabolic interactions within the host's gut which involve immune interactions are also part of the host's immunity and protection by their role in the host metabolism and vitamin synthesis (Kim, 2018; Round \& Mazmanian, 2009; Rowland et al., 2018). Thus, the immune cells participate in the microbial metabolic interactions while contributing to the microbial communities and the host's nutrition. The process of integration considers immunity as these mutual interactions that enable the establishment and maintenance of physiological functions in the host.

The view of holobiont as a host-ecosystem looks at an ecological entity composed of a functioning complex system of energy flow with multiple processes of ecological biotic and abiotic interactions of its organisms as well as molecules and metabolites. I argue that the complex whole of the system of these interactions should be thought of as the immunity of the holobiont that underlies the immunity of each of the interacting organisms (i.e., host and microbes). The interacting organisms do not hold a non-related immunity. Immunity in my view is a shared property of a community within its environmental niche. The collection of all these functions/processes of interactions are taken as the functioning system (Jax, 2005). Thus, if thinking of the process ontology of "things as being derives from processes" (Nicholson \& Dupré, 2018, 13), the immune cells and the immune systems of the host and microbes materializes as the result of the process of their constant interactions with each other and within the environment.

Looking at immunity from an ontological heterogeneity perspective (i.e., multifactorial causation with more than one causal entity) needs to address the question of the immunological entity, i.e., the immunity of what or whom? What is the entity and what is its immunity? My answer to this question is that immunity results from a process of the integration of the interactions between different organisms in their environmental niche into a functioning system. Thus, the immune self or immunological identity should be identified as this functioning (or interacting) system which is not the property of one entity alone but rather materializes through the interactions and intra-actions between many within the holobiont, and also between

\footnotetext{
8 Stability here should be understood in its ecological meaning of fluctuation and constant change (i.e., homeorhetic stability).
} 
holobionts (Barad, 2007; Griesemer, 2014). Immunity in this regard does not belong to one organism but rather a property of the ecological system that the host established with its microbiome.

In the holobiont, the host is both a part of the ecological community (i.e., host and microbes), as well as its spatiotemporal landscape or ecological niche (i.e., host ecosystem). Thus, since the host is one of the organisms in the community but also its ecological niche it is entangled with the holobiont, and so is the host's immunological identity entangled with the holobiont's immunological identity. Thus, I argue that immunity is less of a physiological property per se, but should be studied as the property of the organism's ecological system(s). Continuing this claim, in the next section I will distinguish the definition of the holobiont self (i.e., immunological identity of a host ecosystem) and its implication for understanding boundaries.

\section{The holobiont self: the immunity of an ecological entity}

Boundaries are an important aspect of immunology, their explanatory role is in distinguishing the immunological entity and its protection. But what is the importance of boundaries when thinking about immunity in terms of the process of integration and persistence of interactions?

Boundaries and the demarcation of borders are viewed differently in ecology than the way they are portrayed in immunology. In ecology, borders are flexible and delineated by patterns of different types of interactions and landscapes (Millstein, 2018; Odenbaugh, 2007, 2010). Therefore, in ecology, the boundaries between ecological entities are permeable and sometimes overlapping (McShane, 2004; Millstein, 2018; Odenbaugh, 2010). In the traditional sense of immunology, boundaries are distinctive and necessary for the immune system's function of protection by discrimination. The immune system in its traditional view distinguishes the organism's boundaries by the demarcation of elements in the body as triggers and nontriggers for immune response. However, in viewing the holobiont as a community constructed closely with the host (or as a host ecosystem) an alternative view of boundaries is needed. Thus, the boundaries get a different role from that of establishing belongness or a coherent identity to that of establishing a complex system of interactions (i.e., a functioning system in ecological terms) which contributes to the larger system's immunity (i.e., the integration and persistence of the host's physiological systems and microbiomes).

The delineation of the boundaries of an ecosystem and a community follows the landscape as well as the level and strength of these causal interactions and interdependence (Millstein, 2018; Odenbaugh, 2010). When examining ecosystems, such practice of boundary delineation shows that "ecosystems can exist at different scales and may be embedded in one another as parts to wholes" (Odenbaugh, 2010, p. 245). Thus, by considering Tauber's notion of eco-immunity and my suggestion of the host ecosystem, the holobiont's boundaries should be determined by the different levels of the integration of interactions and not the discrimination between interactions. Also, it can be the case that there are many different microbial communities and ecosystems (i.e., microbiomes) within and around the holobiont and their causal 
interactions under empirical investigation. For example, the vaginal ecosystem and the gut ecosystem are part of a human host ecosystem. Each has its unique microbial community with different dynamical interactions. The vaginal ecosystem is less diverse but changes through the menstruation cycle and the gut ecosystem is diverse but more stable. Also, there are some indications that both ecosystems also experience mutual changes that feedback on each other of microbial composition, body's tissues, and cells, as well as abiotic components of oxygen, metabolites, and acidity. Thus, both ecosystems belong to a holobiont and are connected through the host's systems.

To better understand this perspective regarding the holobiont's borders and immunity, consider this analogy from ecology: a lake is an ecosystem composed of biotic and abiotic elements with a spatiotemporal existence. In this analogy, the lake ecosystem is analogous to the host ecosystem which is the holobiont. Thinking about the lake's existence and continuity, we need to account for all the components and their processes of interactions as well as the changes they undergo through time in their composition. Thus, it will be strange to think of some biotic components as belonging to the lake compared to others just because these components are temporary and interchangeable. Better instead is to understand the dynamic and relations within the lake ecosystem, the intimacy and continuity of the interactions, and their trajectories. In other words, classifying the organisms in an ecosystem in terms of domestic/native/non-pathogenic or foreigner/invasive/pathogenic is limited without the understanding of the interactions dynamic, their place of occurrence, energy flow, and contribution to the ecosystem's capacities. ${ }^{9}$

The organisms in a lake, including the microbial communities, are an essential part of the lake's ecosystem and life cycle with a web of interactions in various degrees of interdependence. Organisms such as bacteria, algae, invertebrates (i.e., plankton, crustaceans, mollusks, and insects), and plants as well as vertebrates are interdependent in trophic interactions and shape the lake's features such as nutrient content, dissolved oxygen, pollutants, $\mathrm{pH}$, and sedimentation. Such features are factors in the lake's 'life cycle' and gradual extinction. ${ }^{10}$ The biotic component can have different intensities of interactions, some are permanent residents such as the algae or microbes and others are constant visitors such as birds, or grazers.

All lakes have a life cycle of different lengths depending on their different features. Nevertheless, the biotic components of the lake are an important part of its processes of stability and persistence and are such across the lake's boundaries. Thus, the boundaries are defined in terms of interactions and not in terms of belonging. If evaluating the lake's state in terms of stability and resilience, then we can use the term beneficial to characterize changes such as migration. Changes in the biota composition such as the migration of species may change the lake's features

\footnotetext{
9 See a similar work by Foster et al. (2017) The evolution of the host microbiome as an ecosystem on a leach. Their model of ecosystem on a leash predicts the evolution of host-microbiome interactions. Thus, their perspective combines both the dynamics of microbial competition and the host's various mechanisms of control (i.e., physiological, behavioral, and immunological) as part of a unique ecosystem that is a living host organism.

${ }^{10}$ For lack of a better term the 'life cycle' refers to the lake's cycle of existence. I am not suggesting here that a lake is a living being.
} 
influencing its state of stability or persistence. Thus, for example, if a migrant species contributes to the lake's stability and persistence over time it benefits the lake and all the organisms in it. If the migrate species causes dramatic decomposition of plants and algae filling the basin with sediment and plant material, it is less beneficial to the lake's stability and persistence.

A distinction between the biotic component that 'belong' to the lake ecosystem and those that are 'visitors' or 'migrants' may be possible in terms of the intensity of the interactions and function in shaping the lake's features. However, such a distinction is not essential in the determination of the lake's state of stability and persistence. The lake's 'immunity,' which in this example is in terms of stability and persistence is the outcome of various interactions in various levels of intimacy and consistency that is also influenced by the abiotic components. ${ }^{11}$ In this regard, the lake's boundaries are important for the understanding of its features and unique interactions. But the criteria of clear inside/outside or belonging/foreign discrimination are not crucial for the definition of the lake's condition rather it is the nature and dynamics of these interactions as they materialized into the lake's functioning system (Jax, 2005; Millstein, 2020a, b). It is more important to look at the levels of integration of species within the ecosystem and their contribution to the 'life cycle' than to determine discrimination criteria. Or in other words, the discrimination criteria of for example invasive vs. migrate species in ecology usually comes after the determination of the nature of the interactions and their function.

Similar to the example of the lake, the immunity of the holobiont is the process of the holobiont's integration and persistence of interactions that contribute to the systemic functioning of the host and its general stability. Therefore, the immune systems of the host and microbes materialized through the immunity of the holobiont. When the host dies, the holobiont soon after changes into a different ecosystem of mostly decomposers (microbes and other detritivores) with different systemic functions. The host is one of the organisms in the holobiont but with a special role in it. The host's special role is similar to the basin of the lake. Once the lake flattens, it is no longer a lake, in this process the ecological community changes accordingly just as the host dies the holobiont features change into a gradual process of decomposing the host until there is no longer a holobiont. Furthermore, in the holobiont ecosystem, the immune interactions include a wider range than the reaction to triggers between tolerance and response. Other types of interactions are essential for

\footnotetext{
11 I put immunity in quotation marks because the concept of immunity or health is contentious in its definition, both when it concerns organisms, e.g., humans, and more so when it comes to ecosystems. The main challenge is in coming up with a satisfying definition that is purely empirical and free of values and normative judgments (Boorse 1977; Costanza 1992; Kingma 2010; Wakefield 1992). Therefore, to avoid the need to commit to any of the suggested definitions and for the sake of my analogy, I consider immunity in terms of stability and persistence as it is usually the case that stability and persistence are better than the lack of persistence and stability (in terms of non-equilibrium cycles). Here, I adopt McShane's suggestion of normative evaluation of healthy vs. unhealthy as the healthy being the preferred state all things considered (McShane 2004). However, the definition of health or immunity needs an additional inquiry in a separate but related paper. Furthermore, I believe that thinking about the holobiont as a host ecosystem can benefit such inquiry, as it is already clear that these notions are very much connected when it comes to questions of health. See for example the concept of one health (Zinsstag et al., 2011) and the notion of population health (Valles 2018).
} 
immunity such as the range of strong and weak inflammation, collaboration and competition between bacterial communities, the collaboration or molecular communication between microbes and immune cells, predation, and so on (Gilbert \& Tauber, 2016; Tauber, 2016; 2017; Chiu \& Eberl, 2016).

One limitation of this analysis is the lack of discussion on the transgenerational stability of immunity, meaning the formation and colonization of the host-microbial communities. It is likely that the colonialization events in a lake ecosystem and a host ecosystem are different mainly due to the host reproduction processes. Also, there are extreme differences in colonization and microbial transmission mechanisms between different holobiont species (e.g., plants, marine invertebrates, mammals). Although, this is an important aspect of the holobiont and other ecosystems in nature it is less relevant to the point of my analogy of understanding boundaries in ecology and the holobiont's physiology. However, I do believe this issue of colonization both in ecology and the holobiont is important for the understanding of macrobe and microbial co-evolutionary processes within complex ecological systems. ${ }^{12}$

Thinking about the holobiont's boundaries in terms of interaction is more helpful when the notion of immunity means stability and persistence through a variety of functions related to different capacities such as metabolism, growth, and reproduction, all of which involve patterns of interactions. The traditional inside-outside distinction should change to inside-outside connections/dynamics and the boundaries understood in terms of outlines that can move and change. The immunity as protecting from harm reflected through these interactions and its meaning is the holobiont's stability (in a non-equilibrium meaning) and persistence of functions through time in its environment. The holobiont self and its immunity as a process of integration of interactions also challenge the war metaphor in immunology. Once immunity is seen as a process of mutual interactions and immunological identity is of a host ecosystem, the meaning of immunity as integration fits better than that of discrimination. The use of metaphoric terms such as surveillance, detection, elimination, tolerance, and targeted-response to describe what the immune system does, relates to one aspect of the interactions pertaining to immunity. Other aspects should include, mutual interactions, population dynamics, functional integration, and collaboration. This way of thinking about immunity opens wide possibilities of understanding immunity and the functions of the immune system that looks into the ecological dynamics of the interactions.

\section{Possible objections}

There are a few possible objections to the view presented here mostly related to biomedical practices. I present three possible objections regarding the perception of immunity and health as the protection of the cohesiveness of the immunological identity. In my response to these objections, I show some black boxes regarding the individualist view, of the self-environment distinction, the definition of infections, and autoimmune diseases. Furthermore, I argue that the view of the

12 I thank the anonymous reviewer for this clarification. 
host-ecosystem and the holobiont self is in line with the general motivation of views such as one health (Zinsstag et al., 2011), population health (Valles, 2018), and extended health (Morar \& Skorburg, 2018), which promote the understanding of the role of the ecological, social, cultural, and political context in the individual's health. The functional explanation of immunity I present here looks at the immunity of the host ecosystem and can contribute to some of the challenges raised regarding these views by looking at the causal explanations for physiological conditions from an ecological perspective of the interactions and feedback loops in differed contextual cycles.

The first, objection argues that connecting the holobiont with the immunity is unnecessary. In this view, we have the host-organism with its immune system forming an immunological unit which includes the interactions with the microbial communities in different levels of dependence. For example, the Squid and the $V$. fischeri are in symbiotic interactions and the notion of immunity refers to the squid, not the squid-bacteria host-ecosystem. Furthermore, if a distinction can be made clear by the immune function and the immunological identity, then it is simpler and more direct to look for the precise discrimination mechanism that allows the immune system to maintain the cohesiveness of the host-microbial interactions.

Such a picture not only helps with the organism's physiological delineation of boundaries. This framing of immunity leads to my second and third objections that fit the causal explanation connecting bacteria or viruses as a cause for infections (e.g., Clostridium difficile, Helicobacter pylori, or SARS-CoV-2) or immunological dysfunction as a cause for chronic non-inflectional diseases. Thus, the one causal direction connecting microbes to disease or looking for a generalization in immune effective response such as immunogenicity is within the paradigm of the discriminatory mechanisms looking for the causal explanations within the organism.

To these objections, my response is that the causal explanations of the role of microbes in infection and immunological dysfunction are already known to be somewhat weak because it ignores the multifunctional causes of the dynamic of interactions and background conditions (Lynch et al., 2020; Schneider, 2020b). For example, in many studies of Inflammatory Bowel Diseases (IBD), the cause of chronic inflammation is unknown and unexplainable by the framework of the discriminatory mechanism and immunogenicity (Morar \& Bohannan 2019; Schneider, 2020a). The view presented here of ontological heterogeneity and the immunity from the holobiont perspective provide a conceptualized framework to new and existing biomedical practices that consider the microbiome as a whole not targeting specific pathogens (e.g., microbiota faecal transplant (MFT), probiotic and prebiotic consumption). The different practical framework looks at the immunological interactions as an ecological environment with checks and balances involve various interactions. Therefore, the therapeutic solutions incorporate assumptions regarding the dynamic of interactions and the changes in the gut's environment as the aim of the therapeutic practices (Morara \& Bohannan 2019). Also, this complexity and ecological terminology (i.e., gut environment) do not fit the common meaning of disease as infection and dysfunction. 
Furthermore, in many cases, the actual disease or harmful symptoms are the results of a combination of compositional and environmental changes in bacterial communities, not an infection/transmission/migration of new species, meaning that the disease occurs in response to already existing microbes that proliferate or changes its pattern of interactions and metabolism. Thus, the change in immune response might be the result of a change in background conditions. In many cases of changes in microbial composition or migration, the infection levels depend also on the dynamics of interactions (and molecular composition) with other microbes present (similar to the ecological community in the lake when migrating species can compete and occupy niches or integrate into the web of interactions already present).

Thus, my response to this objection is that when symptoms of the disease appear, instead of using the terminology of infection and immune response, it is better to look at the dynamic of the environmental interactions within the body and in its surrounding. For example, in many cases of known infectious diseases, the bacteria were already present (e.g., Streptococcus, Salmonella, and are all-ready part of our microbiome) and the symptoms can be a sign that some species become colonizers, take over space, and changes the existing dynamic of interactions and functions. This, of course, comes in degrees depending on the changes and how the various microbial communities and immune cells react to the changes, i.e., in their ability to integrate. This way of thinking also explains the variation of symptoms between individuals with the disease. For example, in the ecological view, treating symptoms of IBD is not only by educating the immune system, but it is also the understanding that the bacterial communities should be 'educated' as well (e.g., MFT is a form of community 'education'). ${ }^{13}$ This perspective also supports the view of extended health which connects medical practices with the individual's close and immediate surroundings (Morar \& Skorburg, 2018).

Finally, the search for a proper explanation of immunogenicity as a framework for diseases that are caused by the immune system such as allergies, arthritis, IBD is also limited. The immune self metaphor within the protection notion of immunity explains these medical conditions with the view that the immune system attacks the body where it should not. Thus, the treatment is by educating the immune system or by suppressing its responses. For example, new cancer treatments are now following the logic of educating the immune system to react to malignant tumors. The same logic works with allergy treatment of desensitizing the immune system. Thus, the objection is that changing the terminology from the war metaphor to integration is trivial and does not help in introducing new ways of understanding such conditions giving rise to new and better methodological practices.

My response is that a disease examined in terms of integration or lack of integration between host and microbes is better in framing such dysfunction or deficiency through the lenses of the dynamic of interactions. Thus, new existing practices of "educating" the immune system as in cancer or educating the microbiome in IBD are better explained within an ecological framework than the immunological discriminatory framework. For example, the dynamic of the interactions between the

\footnotetext{
13 The meaning of the term 'education' refers to the change in the dynamical interactions that will promote integration and not colonialization.
} 
immune cells and the microbial cells in the host can either balance the growth of one microbial species or body cell, or become affected by it and change. Immunodeficiency can be the lack of functions contributing to one or more systems due to changes in the dynamic of the interactions (such as checks and balances of cell growth or gut metabolism). From an ontological heterogeneity view of the host ecosystem, these functions are mutually produced by the interactions with microbes and cells in the host's body.

The future implications of such an understanding of immunity and boundaries are the ecological and environmental understanding of health as well as diseases. The holobiont is a host ecosystem that is part of a larger ecosystem involving other holobionts and microbial communities. Thus, the idea of the immunity of the holobiont involves the processes of the immunity of its surrounding ecosystem. This view can be beneficial in thinking about vaccination, antibiotic resistance, and the food industry by coherently connecting environmental health issues with individual medical conditions. Thus, medical conditions are also addressed in terms of environmental and ecological understanding of the individual holobiont by looking at its host-microbial integration. From this perspective the biomedical therapeutic solutions should be contextual, such as the household and close family suggested by the extended health hypothesis, and also agriculture and food production as suggested by one health concept. ${ }^{14}$

\section{Summary}

I discussed the holobiont challenge to the meaning and understanding of immunity, the host immune system, and the meaning of boundaries regarding individuality. Firstly, I discussed the unique structure of the holobiont as a host ecosystem. Secondly, I discussed the notion of immunity from the holobiont perspective as a process of integration of interactions and persistence of the complex system of interactions (i.e., functioning system). Lastly, I discussed the notion of the holobiont self and the meaning of boundaries in terms of integration of interactions which is different than the traditional role of distinction. Thus, the immune function of separation and discrimination can be thought of as one of many functions under the umbrella of the process and integration of interactions.

In the holobiont literature, the main discourse is on the question of individuality with the three following main definitions of the holobiont as an evolutionary

\footnotetext{
14 The view I am presenting here offers an argument supporting notions of health in context (vs. the emphasis on the individual's health). The emphasis on the context in views such as One Health, Extended Health, and Population Health discusses the importance of different contextual aspects (e.g., social norms, industrial agriculture, environmental conditions, and interactions). My view is in line with the extended health approach, providing an ecological framework for interactions in health. In particular, my view can benefit from the latter's analysis of the close interactions having a constitutive role in individuality. However, I also think that broader contexts should be included, such as social and economic norms and infrastructure, industrial agriculture, and other environmental conditions. Therefore, my view does not need to take a stand on which context is more essential or achieves better practical results, rather it provides an argument for the importance of the environment and the processes of interactions in health in general.
} 
individual (Zilber-Rosenberg \& Rosenberg, 2013), physiological individual (Pradeu, 2016), and ecological community (Skillings, 2016). These three conceptualizations are not generalizations of all forms of holobionts, rather they examine each case or perspective of host-microbial relations. For example, the squid-Vibrio is a case for an ecological community from the microbial perspective or as Lloyd and Wade show a Demibiont (Lloyd \& Wade, 2019; Suárez \& Stencel, 2020). In my analysis, I do not commit to any distinctions between physiological or evolutional individual, or demibiont or holobiont, rather I suggest an ecological framework of individuality that is helpful in the conceptualization of immunity and biomedical practices. This view is wide enough, on the one hand, to include all forms of holobionts as ecological entities from the microbial perspective, and also the immunological perspective of the host, and on the other hand, enables the specificity of scrutinizing each case characteristics by its interactions, microbial functions, degrees of cohesion and possibly evolutionary processes.

In my analysis, I ask how to characterize the holobiont as an immunological entity of host-microbes in ecological terms. This shift also reflects on the notion of the holobiont's boundaries as a dynamic part of the process of interactions. In many ecosystems, as in the example of the lake, the study is of the interactions between the organisms in their communities and their effect on the persistence and stability of the ecosystem functioning whole. Therefore, thinking about the holobiont as a host ecosystem with unique features opens a possibility of an ecological inquiry of the host-organism and its relation to the environment. Then, immunity can be thought of as the complexity of the integration of interactions within the host ecosystem, and immunogenicity as ontologically heterogeneous involving different processes of interactions. Furthermore, this conceptual work benefits the shift in the understanding of the role of the environmental conditions in health and disease, and the therapeutic solutions accordingly.

\section{References}

Baquero, F., Coque, T. M., Galán, J. C., \& Martinez, J. L. (2021). The origin of niches and species in the bacterial world. Frontiers in Microbiology, 12. https://www.frontiersin.org/article/https://doi.org/10. 3389/fmicb.2021.657986.

Barad, K. (2007). Meeting the universe halfway: Quantum physics and the entanglement of matter and meaning (pp. 132-185). Duke University Press.

Boorse, C. (1977). Health as a theoretical concept. Philosophy of Science, 44(4), 542-573.

Chiu, L., \& Eberl, G. (2016). Microorganisms as scaffolds of host individuality: An eco-immunity account of the holobiont. Biology and Philosophy, 31, 819-837. https://doi.org/10.1007/s10539-016-9552-0

Costanza, R. (1992). Toward an operational definition of ecosystem health. In R. Costanza, B. G. Norton, \& B. D. Haskell (Eds.), Ecosystem health: New goals for environmental management (pp. 239256). Washington: Island Press.

Douglas. A. E., \& Werren, J. H. (2016). Holes in the Hologenome: Why host-microbe symbioses are not holobionts. mBio by the American Society for Microbiology, 7, e02099-15. https://doi.org/10.1128/ mBio.02099-15.

Foster, K. R., Schluter, J., Coyte, K. Z., \& Rakoff-Nahoum, S. (2017). The evolution of the host microbiome as an ecosystem on a leash. Nature, 548(7665), 43-51. https://doi.org/10.1038/nature23292 
Gilbert, S., Sapp, J., \& Tauber, A. I. (2012). A symbiotic view of life: We have never been individuals. The Quarterly Review of Biology, 87(4), 325-341.

Gilbert, S., \& Tauber, A. I. (2016). Rethinking individuality: The dialectics of the holobiont. Biology and Philosophy, 31(6), 839-853.

Griesemer, J. R. (2014). Reproduction and the scaffolded development of hybrids. In L. D. Caporeal, J. R. Griesemer, \& W. C. Wimsatt (Eds.), Developing scaffolds in evolution, culture, and cognition (pp. 23-55). The MIT Press.

Griesemer, J. (2016). Reproduction in complex life cycles: Toward a developmental reaction norms perspective. Philosophy of Science, 83, 803-815. https://doi.org/10.1086/687865

Huneman, P. (2014). Individuality as a theoretical scheme. II. About the weak individuality of organisms and ecosystems. Biological Theory, 9, 374-381. https://doi.org/10.1007/s13752-014-0193-8

Jax, K. (2005). Function and 'Functioning' in ecology: What does it mean? Oikos, 111, 641-648.

Kim, C. H. (2018). Immune regulation by microbiome metabolites. Immunology, 154, 220-229. https:// doi.org/10.1111/imm.12930

Kingma, E. (2010). Paracetamol, poison, and polio: Why Boorse's account of function fails to distinguish health and disease. The British Journal for the Philosophy of Science, 61(2), 241-264.

Koch, E. J., Moriano-Gutierrez, S., Ruby, E. G., McFall-Ngai, M., \& Liebeke, M. (2020). The impact of persistent colonization by Vibrio fischeri on the metabolome of the host squid Euprymna Scolopes. Journal of Experimental Biology, 223, jeb212860. https://doi.org/10.1242/jeb.212860

Konopka, A. (2009). What is microbial community ecology? International Society for Microbial Ecology, 3, 1223-1230. https://doi.org/10.1038/ismej.2009.88

Lloyd, E. A. (2001). Units and levels of selection: An anatomy of the units of selection debates. In R. S. Singh, C. B. Krimbas, D. B. Paul, \& J. Beatty (Eds.), Thinking about evolution: Historical, philosophical, and political perspectives (pp. 267-291). Cambridge University Press.

Lloyd, E. A. (2017). Holobionts as units of selection: Holobionts as interactors, reproducers, and manifestors of adaptation. In S. B. Gissis, E. Lamm, \& A. Shavit (Eds.), Landscapes of collectivity in the life sciences. Vienna Series in Theoretical BiologyIn. MIT Press.

Lloyd, E. A., \& Wade, M. J. (2019). Criteria for holobionts from community genetics. Biological Theory, 14, 151-170. https://doi.org/10.1007/s13752-019-00322-w

Lynch, K. E., Parke, E. C., \& O’Malley, M. A. (2020). How causal are microbiomes? A comparison with the helicobacter pylori explanation of ulcers. Biology and Philosophy, 34, 62. https://doi.org/ 10.1007/s10539-019-9702-2

McFall-Ngai, M., Hadfield, M. G., Bosch, T. C. G., et al. (2013). Animals in a bacterial world, a new imperative for the life sciences. PNAS USA, 110, 3229-3236. https://doi.org/10.1073/pnas.12185 25110

McShane, K. (2004). Ecosystem health. Environmental Ethics, 26(3), 227-245.

Millstein, R. L. (2018). Is Aldo Leopold's land community an individual? In O. Bueno, R. Chen, \& M. B. Fagan (Eds.), Individuation, process, and scientific practices (pp. 279-302). Oxford University Press.

Millstein, R. L. (2020a). Functions and functioning in Aldo Leopold's land ethic and in ecology. Philosophy of Science, $87,1107-1118$.

Millstein, R. L. (2020b). Defending a Leopoldian basis for biodiversity: A response to Newman, Varner, and Linquist. Biology and Philosophy, 35(12). https://doi.org/10.1007/s10539-019-9724-9

Moran, N., \& Sloan, D. (2015) The hologenome concept: Helpful or hollow? PLoS Biology, 13(12). https://doi.org/10.1371/journal.pbio.1002311.

Morar, N., \& Skorburg, J. A. (2018). Bioethics and the hypothesis of extended health. Kennedy Institute of Ethics Journal, 28(3), 341-376.

Nicholson, D., \& Dupré, J. (2018). A manifesto for a processual philosophy of biology. In D. Nicholson \& J. Dupré (Eds.), Everything flows: Towards a processual philosophy of biology. Oxford University Press.

Morar Brendan, N., \& Bohannan, J. M. (2019). The conceptual ecology of the human microbiome. The Quarterly Review of Biology, 94(2) 149-175. https://doi.org/10.1086/703582

O’Malley, M. (2014). Philosophy of microbiology. Cambridge University Press.

Odenbaugh, J. (2007). Seeing the forest and the trees: Realism about communities and ecosystems. Philosophy of Science, 74, 628-641. https://doi.org/10.1086/525609

Odenbaugh, J. (2010). On the very idea of an ecosystem. In A. Hazlett (Ed.), New waves in metaphysics. New waves in philosophy (pp. 240-258). Palgrave Macmillan. 
Pradeu, T. (2010). What is an organism? An immunological answer. History and Philosophy of Life Science, 32, 247-267.

Pradeu, T. (2011a). The limits of the self: Immunology and biological identity. Oxford University Press.

Pradeu, T. (2011b). A mixed self: The role of symbiosis in development. Biological Theory, 6, 80-88. https://doi.org/10.1007/s13752-011-0011-5

Pradeu, T. (2016). Organisms or biological individuals? Combining physiological and evolutionary individuality. Biology and Philosophy, 31, 797-817. https://doi.org/10.1007/s10539-016-9551-1

Pradeu, T. (2018). Genidentity and biological processes. In D. Nicholson \& J. Dupré (Eds.), Everything flows: Towards a processual philosophy of biology. Oxford University Press.

Pradeu, T. (2019). Philosophy of biology: Immunology and individuality. eLife, 8, e47384. https://doi. org/10.7554/eLife.47384

Pradeu, T., \& Carosella, E. D. (2006). The self model and the conception of biological identity in immunology. Biology and Philosophy, 21, 235-252. https://doi.org/10.1007/s10539-005-8621-6

Queller, D. C., \& Strassmann, J. E. (2016). Problems of multi-species organisms: Endosymbionts to holobionts. Biology and Philosophy, 31, 855-873. https://doi.org/10.1007/s10539-016-9547-x

Roughgarden, J., Gilbert, S. F., Rosenberg, E., et al. (2018). Holobionts as units of selection and a model of their population dynamics and evolution. Biological Theory, 13, 44-65. https://doi.org/10.1007/ s13752-017-0287-1

Round, J. L., \& Mazmanian, S. K. (2009). The gut microbiota shapes intestinal immune responses during health and disease. Nature Reviews Immunology, 9, 313-323. https://doi.org/10.1038/nri2515

Rowland, I., Gibson, G., Heinken, A., et al. (2018). Gut microbiota functions: Metabolism of nutrients and other food components. European Journal of Nutrition, 57, 1-24. https://doi.org/10.1007/ s00394-017-1445-8

Schneider, T. (2020a). Can we talk about feminist epistemic values beyond gender? Lessons from the gut microbiome. Biological Theory, 15(1), 25-38. https://doi.org/10.1007/s13752-019-00335-5

Schneider, T. (2020b). Microbial activities depending on background conditions. Biology and Philosophy, 35, 23. https://doi.org/10.1007/s10539-020-9740-9

Schneider, T. (2021). Interactions within the holobiont: On the holobiont's interactions of its microorganisms. Philosophy Theory and Practice of Biology, 13, 5. https://doi.org/10.3998/ptpbio.16039257. 0013.005

Skillings, D. (2016). Holobionts and the ecology of organisms: Multi-species communities or integrated individuals? Biology and Philosophy, 31, 875-892. https://doi.org/10.1007/s10539-016-9544-0

Suárez, J., \& Stencel, A. (2020). A part-dependent account of biological individuality: Why holobionts are individuals and ecosystems simultaneously. Biological Reviews, 95, 1308-1324. https://doi.org/ 10.1111/brv. 12610

Tauber, A. I. (1994). The immune self: Theory or metaphor? Cambridge University Press.

Tauber, A. I. (2016). Immunity in context: Science and society in dialogue. Theoria. https://doi.org/10. $1387 /$ theoria. 14560

Tauber, A. I. (2017). Immunity: The evolution of an idea. Oxford University Press.

Tauber, A. I., \& Chernyak, L. (1991). Metchnikoff and the origins of immunology: From metaphor to theory. Oxford University Press.

Theis, K. R., Dheilly, N. M., Klassen, J. L., Brucker, R. M., Baines, F. J., Botch, T. C. G., Cryan, J. F., Gilbert, S. F., Goodnight, C. J., Lloyd, E. A., Sapp, J., Vandenkoornhuyse, P., Zilber-Rozenberg, I., Rozenberg, E., Bordenstein, S. R. (2016). Getting the hologenome concept right: An eco-evolutionary framework for hosts and their microbiomes. mSystems by the American Society for Microbiology 1, e00028-16. https://doi.org/10.1128/mSystems.00028-16.

Triviño, V., \& Suarez, J. (2020). Holobionts: Ecological communities, hybrids or biological individuals? A metaphysical perspective on multispecies systems. Studies of History, Philosopy, Biology, and Biomedical Science. https://doi.org/10.1016/j.shpsc.2020.101323.

Valles, S. (2018). Philosophy of population health: Philosophy for a new public health era. Routledge, pp. $57-78$.

Waddington, C. H. (1957). The strategy of the genes: A discussion of some aspects of theoretical biology. George Allen \& Unwin.

Wakefield, J. C. (1992). The concept of mental disorder: On the boundary between biological facts and social values. American Psychologist, 47(3), 373.

Zilber-Rosenberg, I., \& Rosenberg, E. (2013). The hologenome concept: Human, animal and plant microbiota. Springer, Retrieved from http://link.springer.com/book/10.1007\%2F978-3-319-04241-1. 
Zinsstag, J., Schelling, E., Waltner-Toews, E., \& Tanner, M. (2011). From "one medicine" to "one health" and systemic approaches to health and well-being. Preventive Veterinary Medicine, 101(3-4), $148-156$.

Publisher's Note Springer Nature remains neutral with regard to jurisdictional claims in published maps and institutional affiliations. 